\title{
A Novel Self-Assembling Silane for Abhesive and Adhesive Applications
}

\author{
A. J. Kinloch*, K. T. Tan** \\ Department of Mechanical Engineering, \\ Imperial College London, Exhibition Road, SW7 2BX, London, UK. \\ and \\ J. F. Watts \\ The Surface Analysis Laboratory, \\ School of Engineering, University of Surrey, \\ Guildford, Surrey, GU2 5XH, UK.
}

* Address for correspondence: A.J. Kinloch, a.kinloch@imperial.ac.uk

** Present address: National Institute of Standards and Technology, Building and Fire Research Laboratory, 100 Bureau Drive, Stop 8615, Gaithersburg, Maryland 20899-8615, USA.

\begin{abstract}
The potential benefits from using a novel vinyl-terminated long alkyl-chain self-assembling silane (VTS) have been investigated. A relatively high water contact-angle of $83^{\circ}$ was found for water on the VTS-pretreated soda-lime glass surface. This revealed that the degree of hydrophobicity of this surface was far more pronounced than for the ultrasonically-cleaned glass surface or the $\gamma$-glycidoxypropyltrimethoxy silane (GPS)pretreated glass surface, which gave contact angles of $14^{\circ}$ and $42^{\circ}$, respectively. Further, good agreement between the measured and the theoretical contact angles for a fully adsorbed vinyl-terminated self-assembled monolayer surface implied that the VTS molecules were adsorbed with the vinyl-terminal groups orientated away from the glass surface. Indeed, X-ray photoelectron spectroscopy (XPS) analysis confirmed that the VTS molecules were adsorbed as a monolayer with the vinyl-terminal groups orientated away from the glass surface. Double-cantilever beam joints were prepared using these various pretreated-glass substrates, using an epoxy adhesive, and they were tested employing a fracture-mechanics approach. The adhesive fracture energy, $G_{c}$, of the VTS-pretreated glass/epoxy joints was found to be far lower in value than for the ultrasonically-cleaned joints or the GPS-pretreated joints. The potential applications of this novel long alkyl-chain silane are two-fold: (a) the relatively very poor adhesion of the VTS pretreatment enables this silane to be used as an excellent abhesive layer, i.e. as a release agent, and (b) the presence of reactive vinyl-terminal groups might allow this silane to be activated to form functional groups that may then react with an adhesive, such as an epoxy resin, and so act as an excellent adhesion promoter to increase the durability of the adhesive joint.
\end{abstract}

Keywords: Abhesion, accelerated testing, durability, glass, monolayers, organosilane adhesion promoters, XPS 


\section{Introduction}

Commercially-available short alkyl-chain silane primers have been used extensively to decrease the susceptibility of the adhesive-substrate interface to attack by water, see for example [1-3]. The ability of these silanes to promote enhanced adhesion to metallic and ceramic substrates is a result of the specific interaction, via a condensation reaction, between the silanols of the hydrolysed silane with hydroxyl groups present on the surface of the substrate to form covalent bonds [1-2]. Typically there is also a functional terminalgroup at the other end of the silane molecule which may react with the adhesive. Thus, silanes may 'couple’, via covalent bonding, the substrate to the adhesive.

However, such commercially-available silane primers have a relatively short alkylchain in their chemical structure, and typically adsorb onto the surface of the substrate as disordered multilayer layers. For example, $\gamma$-glycidoxypropyltrimethoxysilane (GPS) typically adsorbs with approximately $50 \%$ of the silanol groups pointing upwards and 50 \% pointing downwards [4], and typically forms a multilayer polysiloxane structure consisting of between five to twenty-five molecular layers [5]. Thus, although these multilayer silane primers may indeed form strong covalent bonds across the adhesive/substrate interface, the presence of a relatively thick and weak polysiloxane layer (a) may lead to a cohesive failure in the polysiloxane, and (b) still leads to water being able to permeate through the polysiloxane and so reach the silane/substrate interface, where it may disrupt even primary interfacial bonding [6]. Therefore, the silane performance might be significantly improved if the silane could be adsorbed as a highly-ordered monolayer film. Indeed, for example, in the manner of a self-assembled monolayer (SAM), in such a way that the alkyl-chains are densely packed together and orientated, so making any reactive terminal-groups available for reaction with an adhesive. Consequently, this would result in the formation of a covalently-bonded 'bridge' between the substrate and the adhesive, but now without the presence of a relatively thick layer of polysiloxane. Also, in a long alkyl-chained silane, the intermediate alkyl-chains would be highly hydrophobic, as well as relatively close-packed. Therefore, an ordered monolayer film would impede water diffusing through such a SAM-silane layer to the silane/substrate interface. All of these features should help to impart excellent joint durability. 
To enable silane molecules to form a monolayer film, and self-assemble on substrates in such a way that the alkyl chains will be densely packed together and be orientated away from the substrates, it has been demonstrated [7,8] that a critical minimum alkyl chain length of about eighteen and a particular substrate-head group combination are required. The objective of the present research was to explore the potential benefits of using such a self-assembling monolayer silane. A novel vinyl-terminated long alkyl-chain selfassembling silane, with twenty alkyl groups in the chain, was studied. Contact angle measurements and X-ray photoelectron spectroscopy have been undertaken to investigate the details of the silane adsorption. In addition, linear-elastic fracture-mechanics tests have been conducted using adhesively-bonded double-cantilever beam specimens to assess the toughness and durability of the silane-pretreated joints.

\section{Experimental}

\subsection{Materials}

The novel long alkyl-chain silane investigated was 22-triethoxysilyldocos-1-enesilane, i.e. a vinyl-terminated long alkyl-chain silane (VTS). A $\gamma$-glycidoxypropyltrimethoxysilane (GPS) was also investigated for comparative purposes. The schematic chemical structures of the two silanes are shown in Figure 1. The VTS was synthesized by Target Molecules Ltd. (Southampton, UK), whilst the GPS was supplied by OSI Specialities (Wilton, CT, USA). The adhesive used was a hot-curing rubber-toughened epoxy adhesive, which was based upon a dicyandiamide-cured diglycidyl ether of bisphenol-A. The product grade for this adhesive was 'XD ${ }^{\circledR} 4236-2$ ', from Huntsman Advanced Materials (Duxford, UK). The substrates used throughout the present work were manufactured from toughened soda-lime glass. (Glass substrates were used to adsorb the long alkyl-chain silane, since the glass surfaces are relatively smooth which should assist the silane to self-assemble and thereby form an ordered monolayer film.) The bulk composition of the glass is given in Table 1.

\subsection{Specimen preparation}

The adhesively-bonded double-cantilever beam (DCB) test geometry was employed, and is schematically illustrated in Figure 2. The silane solution consisted of $1 \% \mathrm{w} / \mathrm{w}$ of silane and 
$99 \% \mathrm{w} / \mathrm{w}$ of solvent, which was a mixture of $90 \% \mathrm{w} / \mathrm{w}$ ethanol and $10 \% \mathrm{w} / \mathrm{w}$ deionised water. The solution was stirred continuously for one hour to allow the silane to hydrolyse. Meanwhile, the glass substrates were first cleaned with acetone to remove gross contamination before being cleaned ultrasonically in a bath of deionised water. In the case of the silane-pretreated substrates, immediately after the silane solution was ready, the glass substrates were dipped into the solution for 10 seconds. The substrates were then rinsed with the solvent used to prepare the silane solution. The silane-pretreated glass substrates were then dried at $93{ }^{\circ} \mathrm{C}$ for one hour and allowed to cool to room temperature, before being bonded to form the DCB joint. Prior to joint preparation, a thin layer of polytetrafluoroethylene film was laid onto one of the glass substrates, in order to create a starter crack. Typically this extended about $40 \mathrm{~mm}$ from the loading end. Meanwhile, the adhesive was degassed in a vacuum oven at $80{ }^{\circ} \mathrm{C}$ before being applied to the substrates. The bond-line thickness of the adhesive was controlled during manufacture using $0.5 \mathrm{~mm}$ diameter wire inserts. Finally, the joints were held in a purposed-built jig and the adhesive was cured at $130^{\circ} \mathrm{C}$ for three hours.

\subsection{Material characterisation}

Contact angle measurement

Contact angles formed by droplets of deionised water were measured on the various pretreated glass substrates using the sessile drop method, employing a 'Ramè-Hart (Netcong, NJ, USA)100-00’ goniometer.

\section{$X$-ray photoelectron spectroscopy}

X-ray photoelectron spectroscopy (XPS) analyses were undertaken using a 'Thermo VG Scientific (East Grinstead, UK) Sigma Probe' spectrometer. The X-ray source used was monochromatic aluminium Ka set to a spot size of $500 \mu \mathrm{m}$. Survey spectra were performed with a pass energy of $150 \mathrm{eV}$ to detect all peaks, which was followed by a high-resolution examination using a pass energy of $50 \mathrm{eV}$. 


\section{Fracture-mechanics tests}

The DCB joints were tested under monotonic-loading conditions using a screw-driven Instron tensile-testing machine, in compliance with the relevant test standard, BS 7991 [9]. The tests were conducted at a displacement rate of the cross-head of the Instron (High Wycombe, UK) machine of $10 \mu \mathrm{m} / \mathrm{min}$. The test environment was water at $21 \pm 1^{\circ} \mathrm{C}$. The value of $G_{C}$ was ascertained from the following equation:

$$
G_{C}=\frac{3 P u}{2 B(a+|\Delta|)} \cdot \frac{F}{J}
$$

where $P$ is the critical load, $B$ is the width of specimen, $a$ is the crack length, $J$ is a loadblock correction, $F$ is a large displacement correction, $u$ is the displacement and $\Delta$ is a crack-length correction for a beam that is not perfectly built-in [9].

\section{Results and discussion}

\subsection{Contact angle measurements}

The contact angle of water on the VTS-pretreated surface is shown in Figure 3. As described above, for comparative purposes, two different surface pretreatments, i.e. an ultrasonic-cleaning and a GPS pretreatment, were also investigated. It is noteworthy that the contact angle for the ultrasonically-cleaned glass was $14^{\circ} \pm 3^{\circ}$, and was the lowest amongst all the surfaces. This is attributed to the fact that the glass surface is of a highly polar nature, and therefore water molecules are able to wet readily the ultrasonicallycleaned surface. (However, it should be noted that the contact angle was not zero. We would suggest that this is due to relatively low concentrations of adventitious hydrocarbon contamination which adsorbs onto the cleaned glass surface, either from the wash solutions employed or from the atmosphere after cleaning. This suggestion is confirmed by the results from the XPS studies, as shown and discussed below.)

After the adsorption of the GPS film, the contact angle of water on the surface increased significantly and was $42^{\circ} \pm 1^{\circ}$, indicating that the nature of the surface was now more hydrophobic than the ultrasonically-cleaned surface. Such an increase in the contact 
angle by nearly a factor of four is due, of course, to the presence of hydrophobic organic functional groups on the GPS-pretreated glass surface.

Turning to the VTS-pretreated glass, as can be seen from Figure 3, the extent of the hydrophobic nature of the VTS-pretreated surface was much more pronounced than for the GPS-pretreated and the ultrasonically-cleaned glass surfaces, with a very high contact angle of $83^{\circ} \pm 3^{\circ}$. Now, the theoretical contact angle for a self-assembled monolayer silane surface, with the vinyl-terminated orientated away for the glass surface, may be estimated using the Young-Dupré Equation:

$$
W_{a}=\gamma_{l v}(1+\cos \theta)
$$

where $W_{a}$ is the thermodynamically reversible work of adhesion, $\theta$ is the contact angle and $\gamma_{V v}$ is the surface energy of water. For solely dispersion-force interactions, the relationship between the value of $W_{a}$ and the surface energies of water, $\gamma_{v v}$, and a solid surface, $\gamma_{s v}$, may be determined via a geometric mean approach as:

$$
W_{a}=2 \sqrt{\gamma_{l v} \gamma_{s v}}
$$

Combining equations (2) and (3) yields:

$$
\cos \theta=2 \sqrt{\frac{\gamma_{s v}}{\gamma_{l v}}}-1
$$

The values of $\gamma_{l v}$ and $\gamma_{s v}$ for water and a fully adsorbed vinyl-terminated self-assembled monolayer surface have been reported to be $72 \mathrm{~mJ} / \mathrm{m}^{2}$ and $26 \mathrm{~mJ} / \mathrm{m}^{2}$ [10], respectively. These values yield a theoretical contact angle for a fully adsorbed vinyl-terminated selfassembled monolayer surface of $78^{\circ}$. This value is clearly in good agreement with the measured contact angle of $83^{\circ} \pm 3^{\circ}$. Therefore, it is evident that for the VTS-pretreated 
glass surface that all, or least a large majority, of the vinyl-terminal groups were orientated away from the glass surface.

\subsection{X-ray photoelectron spectroscopy studies}

X-ray photoelectron spectroscopy (XPS) has been employed to determine the elemental compositions of the ultrasonically-cleaned and the silane-pretreated glass surfaces. The XPS survey spectra and the elemental composition are shown in Figures 4-6 and Table 2, respectively.

Upon examining the results for the ultrasonically-cleaned surface, see Figure 4 and Table 2, it can be seen that silicon and oxygen were detected at relatively high intensities. The detection of a high intensity of oxygen is to be expected, as it is a major component of the soda-lime glass. Sodium and calcium at relatively low intensities were also detected. These elements are also the main chemical constituents of the soda-lime glass, see Table 1. Interestingly, tin was detected on both sides of the sample. It should be noted that soda-lime glass is made by a float-glass process, which involves melting glass and feeding a thin layer of the molten glass onto a tank of molten tin, protected by an inert nitrogen atmosphere. Therefore, the soda-lime glass surfaces naturally contain some tin, presumably as dissolved $\mathrm{SnO}$ that was derived from the manufacturing process. Also, clearly, the upper and lower surfaces of the soda-lime glass do not experience the same environment during the forming operation. This results in the 'bottom surface' containing a significantly higher concentration of tin, as compared to the 'upper' surfaces [11]. This obviously explains the different concentrations of tin detected on the two sides of the glass samples. (Note: the side of the glass with a lower concentration of tin will be denoted as 'side 1' and vice versa.)

The XPS survey spectra and the elemental composition of the GPS-pretreated sample are depicted in Figure 5 and Table 2, respectively. The adsorption of the silane onto the glass surface is indicated by the slightly higher concentrations of silicon and oxygen as compared to the simply ultrasonically-cleaned glass surface. Also, the intensity of the calcium signal (which originates from the glass) detected on the GPS-pretreated surface 
was relatively low compared to the intensity detected on the ultrasonically-cleaned surface. This implies that an organic overlayer was indeed deposited on the glass surface by the GPS pretreatment. The carbon concentration at 27 to $30 \%$ is very close to that recorded in the study of GPS on hydroxylated iron surfaces [12] and it is thus reasonable to assume that the thickness of the GPS layer is comparable at approximately $1.7 \mathrm{~nm}$. This is somewhat thicker than the length of a hydrolysed GPS molecule which is $1.3 \mathrm{~nm}$. Hence, it appears that the GPS layer exists as a relatively disorganised layer, with no dominant orientation or structuring [12]. The surface concentration of carbon on the GPS-pretreated glass is significantly lower than that for the ultrasonically-treated glass substrate, which on first sight seems counterintuitive, given the discussions above relating to the deposition of the GPS layer. However, this is not the case; on interaction with the glass the GPS molecules will displace the adventitious hydrocarbon contamination which is replaced by a more securely bound, but thinner at about $1.7 \mathrm{~nm}$, layer of the organosilane.

Also, it is noteworthy that a higher intensity of tin was detected on the GPSpretreated surface than on the ultrasonically-cleaned glass surface. This suggests that the tin, which was initially present on the outermost glass surface, has diffused into the polysiloxane layer on the GPS-pretreated glass surface. A similar observation has been reported by Ritter et al. [13].

Comparing the XPS survey spectra for the ultrasonically-cleaned sample and the VTS-pretreated sample (see Figures 4 and 6), the adsorption of the long alkyl-chain silane on the glass surface is shown by an increase in the intensity of the carbon signal and a decrease in oxygen intensity. The detection of relatively low intensities of the sodium and calcium signals also indicates the presence of the organic silane film on the surface. The shape and intensity of the scattered electron background to a higher binding energy associated with the glass-forming elements $\mathrm{Si}, \mathrm{Na}, \mathrm{O}$ and $\mathrm{Sn}$, as shown in Figure 6, indicates more energy loss via inelastic scattering of electrons, compared to Figure 4. Such a phenomenon is most clearly seen in the spectrum of Figure 6(b) where the regions of the spectrum at a higher binding energy (to the left) of the O1s, OKLL, Si2p and Si 2s peaks exhibit a much greater intensity than the spectra of Figures 4 and 5 . This additional electron 
intensity is the result of the inelastic scattering of the photoelectrons emanating from the glass in the thicker, uniform organic overlayer (of VTS). This shows that the silanepretreated surfaces have a thicker layer of organic material present than the ultrasonicallycleaned glass surface. Using the Beer-Lambert principle [14], the thickness of the silane layer was estimated to be $3.0 \mathrm{~nm}$. However, the theoretical molecular length of the fully orientated VTS molecule is $3.4 \mathrm{~nm}$, and thus this may imply that the silane molecules were absorbed as a monolayer but at an angle to the glass surface. A simple geometric approach indicates that the molecules are oriented at approximately $60^{\circ}$ to the plane of the substrate. On the face of it, this is a surprising observation as XPS studies of the adsorption of GPS on iron surfaces have shown that the layer thickness may be somewhat greater than the length on the hydrolysed silane molecule [12]. However the reliability of XPS as a means of assessing the orientation of molecules at solid surfaces is well established and has been used to good effect on "practical" systems. For example, the validation of this approach by using molecular dynamics and XPS analysis of an adhesion failure surface has shown it to be robust and adaptable [15]. Indeed, the fact that the VTS molecules are oriented at approximately $60^{\circ}$ to the plane of the substrate has been reported by previous experimental [7] and theoretical studies [8]. Finally, the loss structures associated with the VTS layer discussed above show that the layer is not present as a patchy overlayer, which would not provide such intense energy-loss structures, giving additional security to the interpretation of the data as a monolayer of VTS molecules in a slanting, rather than normal, orientation.

The spectra of C1s signal were fitted with three peaks corresponding to the hydrocarbon (C-C) at $285.0 \mathrm{eV}$ with the hydroxyl/ether (C-O) at a shift of $1.6 \mathrm{eV}$ relative to the hydrocarbon peak, and the carbonyl/amide $(\mathrm{C}=\mathrm{O}$ or $\mathrm{N}-\mathrm{C}=\mathrm{O})$ at a shift of $3.0 \mathrm{eV}$. The spectrum of the ultrasonically-cleaned class, see Figure 7 , is indicative of the usual adventitious hydrocarbon contamination; with the components at $285.0 \mathrm{eV}$ representing the apolar methylene-type contamination, whilst the other higher binding-energy components represent the oxygen-containing polar contamination. Turning now to the $\mathrm{C} 1 \mathrm{~s}$ spectra of the silane-treated glass surfaces, see Figures 8 and 9, it is obvious that the $\mathrm{C}-\mathrm{O}$ and $\mathrm{C}=\mathrm{O}$ peaks detected on the VTS-pretreated surface were relatively less intense than for the GPSpretreated surface. This observation was indeed expected if the VTS molecules were 
adsorbed as a monolayer film, and thus confirms the conclusion that the VTS film formed on the glass surface was a monolayer, with the vinyl-terminal groups orientated away from the substrate and the molecules pointing upwards at an angle of about $60^{\circ}$ to the glass substrate.

\subsection{Fracture-mechanics tests}

In order to assess the adhesion properties of the various surface pretreatments, constant displacement-rate tests based, upon a fracture-mechanics approach, were undertaken, as described above. Figure 10 summarises the values of the adhesive fracture energy, $G_{C}$, for the various glass/epoxy/glass DCB joints.

It is apparent that the GPS-pretreated joints exhibited a significantly better performance than the ultrasonically-cleaned joints. This observation is in agreement with published work [13, 16-18], where the use of such silane-coupling agents has been widely reported to enhance the interfacial adhesion between the glass substrate and the adhesive. The superior durability performance of the GPS-pretreated joints is attributed to the formation of covalent bonds between the silane coupling agent and the glass surface, and between the silane coupling agent and the adhesive $[1,2,5]$. Thus, the ingressing moisture at the crack tip must now rupture covalent $-\mathrm{Si}-\mathrm{O}-\mathrm{Si}-$ bonds, rather than solely the secondary bonds that act across the adhesive/substrate interface of the ultrasonically-cleaned joints. This results in crack propagation in the GPS-pretreated joints occurring at relatively high values of $G_{\mathrm{C}}$.

It may be seen from Figure 10 that the values of $G_{C}$ ascertained for the joints where the substrates were pretreated with the VTS were relatively very low, compared to the values for the ultrasonically-cleaned joints and the GPS-pretreated joints. This is as expected and arises from the relatively poor wetting and intrinsic adhesion between the epoxy and the vinyl-terminal groups of the VTS-silane, since such vinyl groups are unreactive with respect to the epoxy and are also apolar, as discussed above. 
The very low values of $G_{C}$ may lead to this novel long alkyl-chain silane finding applications as an abhesive coating. However, on the other hand, an advantage of the vinylterminal groups is that it may be possible to 'activate' these functional groups so that they are then able to chemically react with an epoxy adhesive to form covalently bonds between the adhesive and substrate. As commented above, also, in a long alkyl-chained silane, the intermediate alkyl-chains would be highly hydrophobic, so such an ordered monolayer film would impede water diffusing through the monolayer to the silane/substrate interface. All of these features should help to impart excellent joint durability. This work is currently in progress.

\section{Conclusions}

The use of a novel vinyl-terminated long alkyl-chain self-assembling silane (VTS) with a twenty alkyl-chain length has been assessed as a primer for epoxy/glass joints. A relatively high contact angle of $83^{\circ}$ was found for water on the VTS-pretreated soda-lime glass surface. This revealed that the degree of hydrophobicity of this surface was far more pronounced than for the ultrasonically-cleaned glass surface or the $\gamma$ glycidoxypropyltrimethoxy silane (GPS)-pretreated glass surface, which gave contact angles of $14^{\circ}$ and $42^{\circ}$, respectively. Further, good agreement between the measured and the theoretical contact angles for a fully adsorbed vinyl-terminated self-assembled monolayer surface implied that the VTS molecules were adsorbed with the vinyl-terminal groups orientated away from the glass surface. Indeed, X-ray photoelectron spectroscopy (XPS) analysis confirmed that the VTS molecules were adsorbed as a monolayer with the vinylterminal groups orientated away from the glass surface. XPS also revealed that the adsorbed VTS molecules which formed the monolayer were inclined at an angle of about $60^{\circ}$ to the glass surface.

These conclusions were borne out by the results from the fracture-mechanics tests, where the adhesive fracture energy, $G_{C}$, for the VTS-pretreated glass-epoxy-glass joints was relatively very low compared to the ultrasonically-cleaned joints and the GPS- 
pretreated joints. The very low values of $G_{C}$ for the VTS-pretreated joints may lead to this novel long alkyl-chain silane finding applications as an abhesive coating. However, on the other hand, an advantage of the vinyl-terminal groups is that it may be possible to 'activate' these functional groups so that they are then able to chemically react with an epoxy adhesive to form covalently bonds between the adhesive and substrate. Also, in a long alkyl-chained silane, the intermediate alkyl-chains would be highly hydrophobic, so such an ordered monolayer film would impede water diffusing through the monolayer to the silane/substrate interface. All of these features should help to impart excellent joint durability. This work is currently in progress.

\section{Acknowledgements}

The authors would like to thank Mr. J.A. Bishopp for useful advice on the use of the long alkyl-chain silane and Dr. M-L. Abel for the calculation of silane-layer thickness. The assistance of Mr. S. Greaves of University of Surrey in undertaking the XPS analyses is gratefully acknowledged. Also, the authors would like to thank the UK Overseas Research Scholarship Office and Dr. Dave Tod at QinetiQ, UK, for sponsoring Dr. K.T. Tan. 


\section{References}

[1] Getting, M., and Kinloch, A. J., J. Mater. Sci. 12, 2511-2518 (1977).

[2] Abel, M-L., Watts, J. F., and Digby, R. P., J. Adhes. 80, 291-312 (2004).

[3] Pleuddemann, E. P., Silane Coupling Agents (Plenum Press, New York, 1991).

[4] van Ooij, W. J., Zhang, B. C., and Conners, K. D., Am. Inst. Phys. 33 (6), 11621170 (1996).

[5] Kinloch, A. J., Proc. Inst. Mech. Engrs. Part G 211, 307-335 (1997).

[6] Bascom, W. D., Macromolecules 5, $792-798$ (1972).

[7] Cave, N. G., and Kinloch, A. J., Polymer 33 (6), 1162-1170 (1992).

[8] Hobbs, P. M., and Kinloch, A. J., J. Adhes. 66, 203-228 (1998).

[9] British Standard 7991, Determination of the mode I adhesive fracture energy $G_{I C}$ of structure adhesives using the double cantilever beam and tapered double cantilever beam specimens (2001).

[10] Collet, J., and Vuillaume, D., Appl. Phys. Lett., 73 (18), 2681-2683 (1998).

[11] Ernsberger, F.M., Annual Reviews, 529-572 (1972).

[12] Davis, S. J., and Watts, J. F., Int. J. Adhes. Adhes. 16, 5-15 (1996).

[13] Ritter, J. E., Learned, J. C., Jacome, G.S., Russell, T. P., and Lardner, T. J., J. Adhes. 76, 335-351 (2001).

[14] Watts, J. F., and Wolstenholme, J., An Introduction to Surface Analysis by XPS and AES (John Wiley and Sons, Chichester, 2003).

[15] Taylor, A. M., McLean, C. H., Charlton, M., and Watts, J. F., Surf. Interface Anal. 23 (5), 342-348 (1995).

[16] Ritter, J. E., Fox, J. R., Hutko, D. I., and Lardner, T. J., J. Mater. Sci. 33, 4581-4588 (1998).

[17] Ritter, J. E., Jacome, G. S., Pelch, J. R., Russell, T. P., and Lardner, T. J., ASME J. Electron. Packag. 124, 328-333 (2002).

[18] Ritter, J. E., and Huseinovic, A., ASME J. Electron. Packag. 123, 401-404 (2001). 
Table 1. Bulk composition of the toughened soda-lime glass.

\begin{tabular}{|l|c|c|}
\hline Materials & Chemical formula & Composition (\%) \\
\hline Sand & $\mathrm{SiO}_{2}$ & 72.8 \\
\hline Soda ash & $\mathrm{Na}_{2} \mathrm{O}$ & 13.0 \\
\hline Limestone & $\mathrm{CaO}$ & 8.4 \\
\hline Dolomite & $\mathrm{MgO}$ & 4.0 \\
\hline Alumina & $\mathrm{Al}_{2} \mathrm{O}_{3}$ & 1.0 \\
\hline Other & - & 0.8 \\
\hline
\end{tabular}


Table 2. Surface composition (atomic \%) for the various glass substrates.

\begin{tabular}{|c|c|c|c|c|c|c|}
\hline & \multicolumn{2}{|c|}{ Ultrasonic cleaning } & \multicolumn{2}{c|}{ GPS } & \multicolumn{2}{c|}{ VTS } \\
\hline Elements & Side 1 & Side 2 & Side 1 & Side 2 & Side 1 & Side 2 \\
\hline $\mathbf{C}$ & 38.8 & 38.4 & 27.0 & 29.8 & 54.0 & 64.0 \\
\hline $\mathbf{C a}$ & 1.4 & 1.6 & 1.1 & 1.1 & 0.9 & 0.8 \\
\hline $\mathbf{N}$ & 1.0 & 0.8 & 0.3 & 0.4 & - & 0.5 \\
\hline $\mathbf{N a}$ & 1.6 & 2.4 & 3.2 & 1.9 & 1.7 & 1.0 \\
\hline $\mathbf{O}$ & 42.5 & 42.4 & 49.4 & 44.6 & 30.6 & 24.1 \\
\hline $\mathbf{S i}$ & 13.9 & 11.8 & 15.8 & 12.5 & 11.8 & 8.3 \\
\hline $\mathbf{S n}$ & 0.6 & 2.6 & 3.2 & 9.7 & 0.6 & 1.3 \\
\hline $\mathbf{M g}$ & 0.2 & - & - & - & - & - \\
\hline $\mathbf{A l}$ & - & - & - & - & 0.4 & - \\
\hline
\end{tabular}




\section{Figure Captions}

Figure 1. Structures of (a) the VTS and (b) the GPS.

Figure 2. The DCB adhesive joint. The thickness of the adhesive layer was $0.5 \mathrm{~mm}$ and the length of the substrate beam was $140 \mathrm{~mm}$.

Figure 3. Contact angles of water on the various pretreated glass substrates. The error bars represent the standard deviations.

Figure 4. XPS survey spectra of the ultrasonically-cleaned samples for (a) side 1 and (b) side 2.

Figure 5. XPS survey spectra of the GPS-pretreated samples for (a) side 1 and (b) side 2.

Figure 6. XPS survey spectra of the VTS pretreated samples for (a) side 1 and (b) side 2.

Figure 7. High-resolution XPS spectra of the peak-fitted C1s signal for the ultrasonically-cleaned glass; (a) side 1 and (b) side 2.

Figure 8. High-resolution XPS spectra of the peak-fitted C1s signal for the GPSpretreated glass; (a) side 1 and (b) side 2 .

Figure 9. High-resolution XPS spectra of the peak-fitted C1s signal for the vinylterminated long carbon-chain silane(VTS)-pretreated glass; (a) side 1 and (b) side 2 .

Figure 10. Values of the adhesive fracture energy, $G_{C}$, for various types of the glass/ epoxy/glass DCB joints tested in an aqueous environment. The crack velocity for these tests was $0.3 \mathrm{~mm} / \mathrm{min}$. The error bars represent the standard deviations. 
(a)<smiles>C=CCC(C)[Si](OCC)(OCC)OCC</smiles>

(b)<smiles>CO[Si](C)(OC)C(C)OCC1CO1</smiles>

Figure 1. Structures of (a) the VTS and (b) the GPS. 


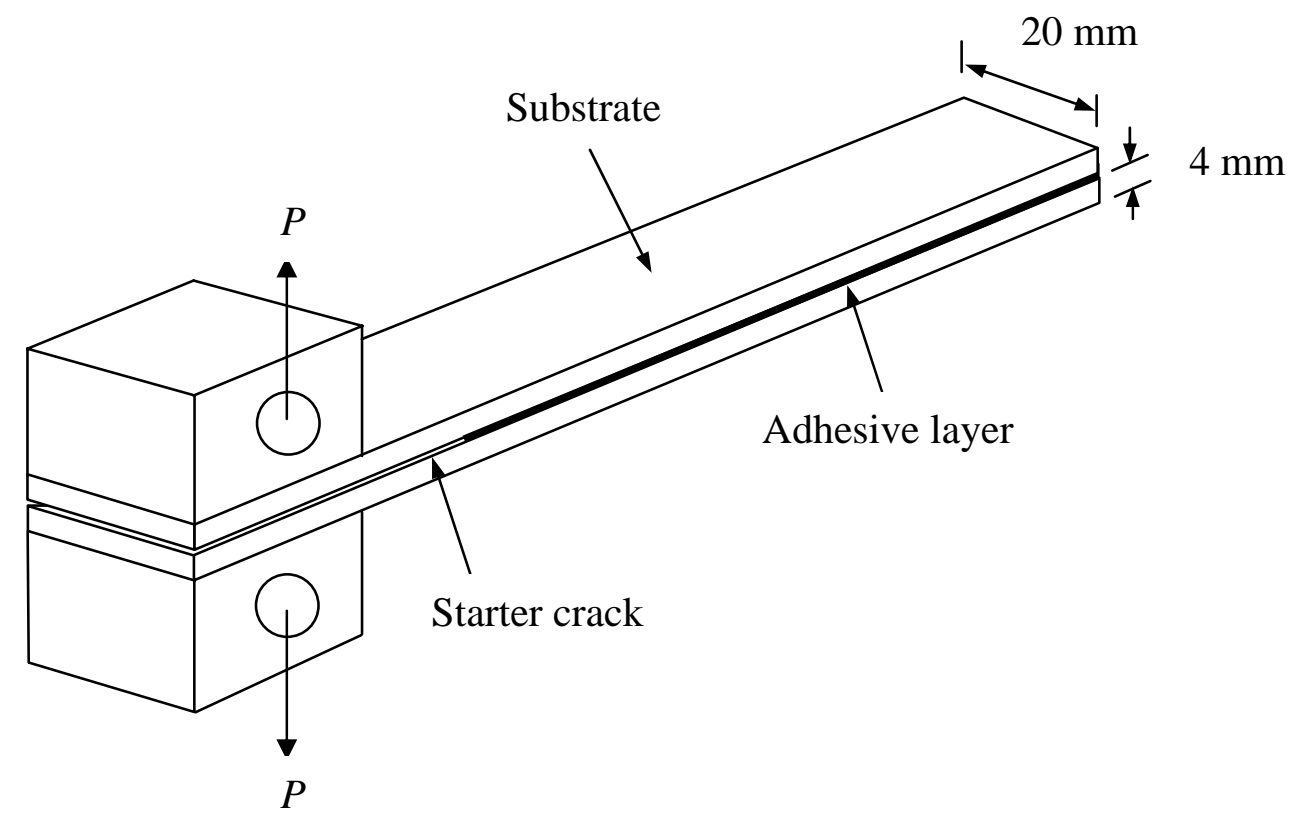

Figure 2. The DCB adhesive joint. The thickness of the adhesive layer was $0.5 \mathrm{~mm}$ and the length of the substrate beam was $140 \mathrm{~mm}$. 


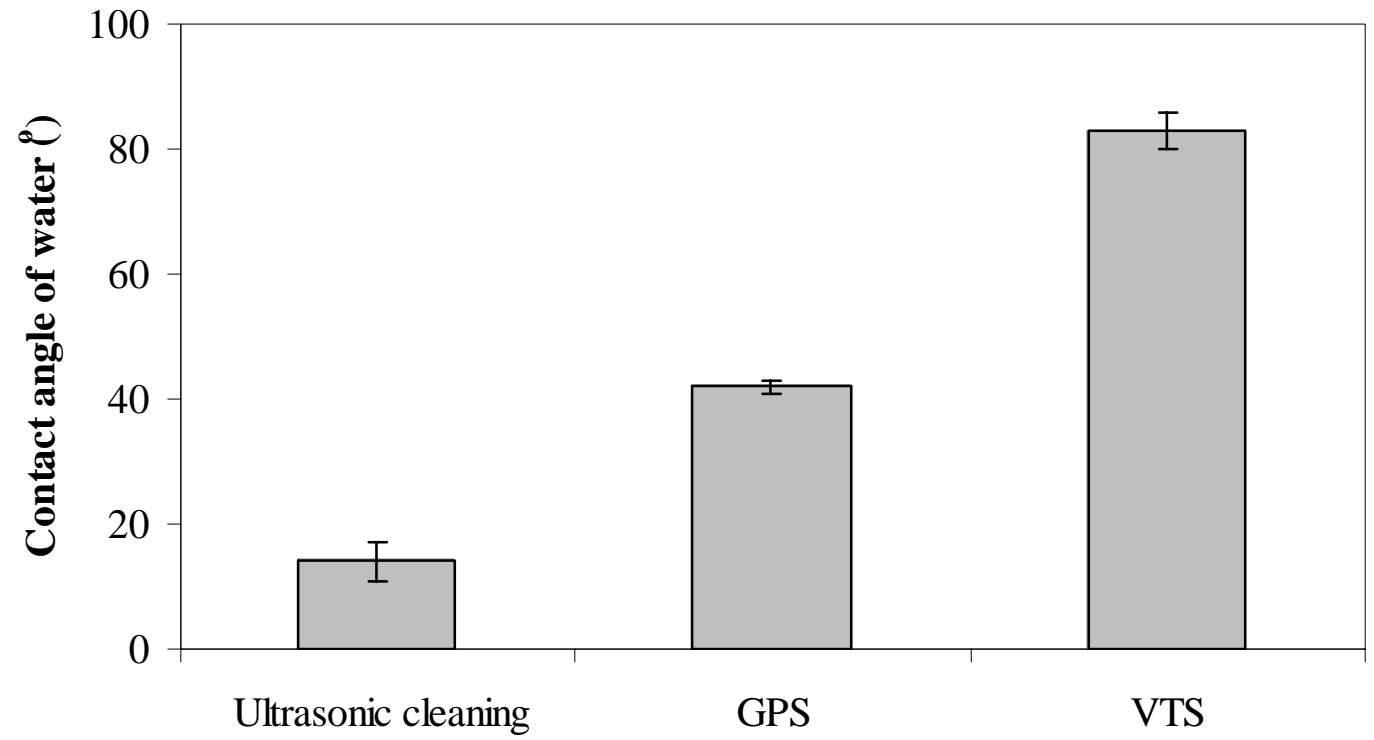

Figure 3. Contact angles of water on the various pretreated glass substrates. The error bars represent the standard deviations. 
(a)

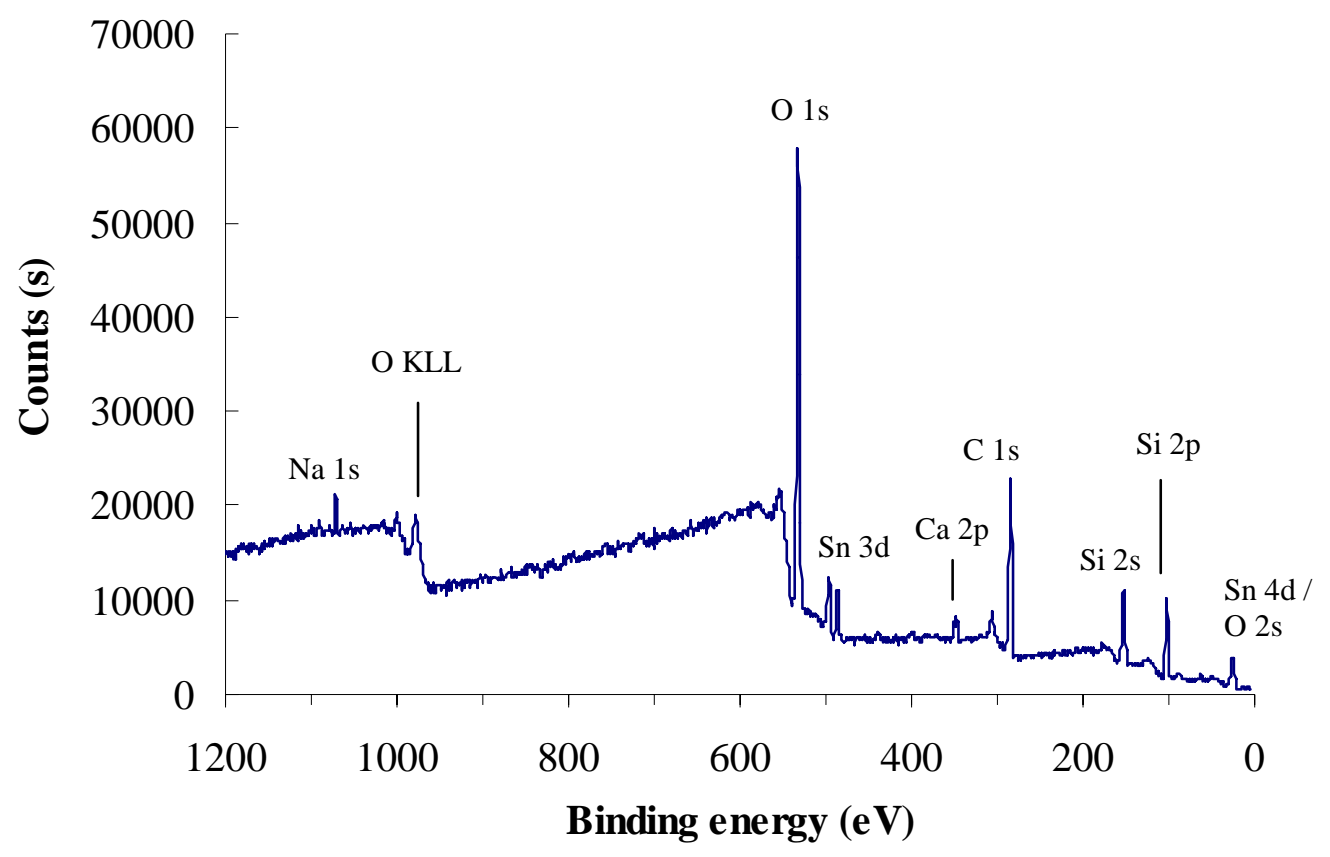

(b)

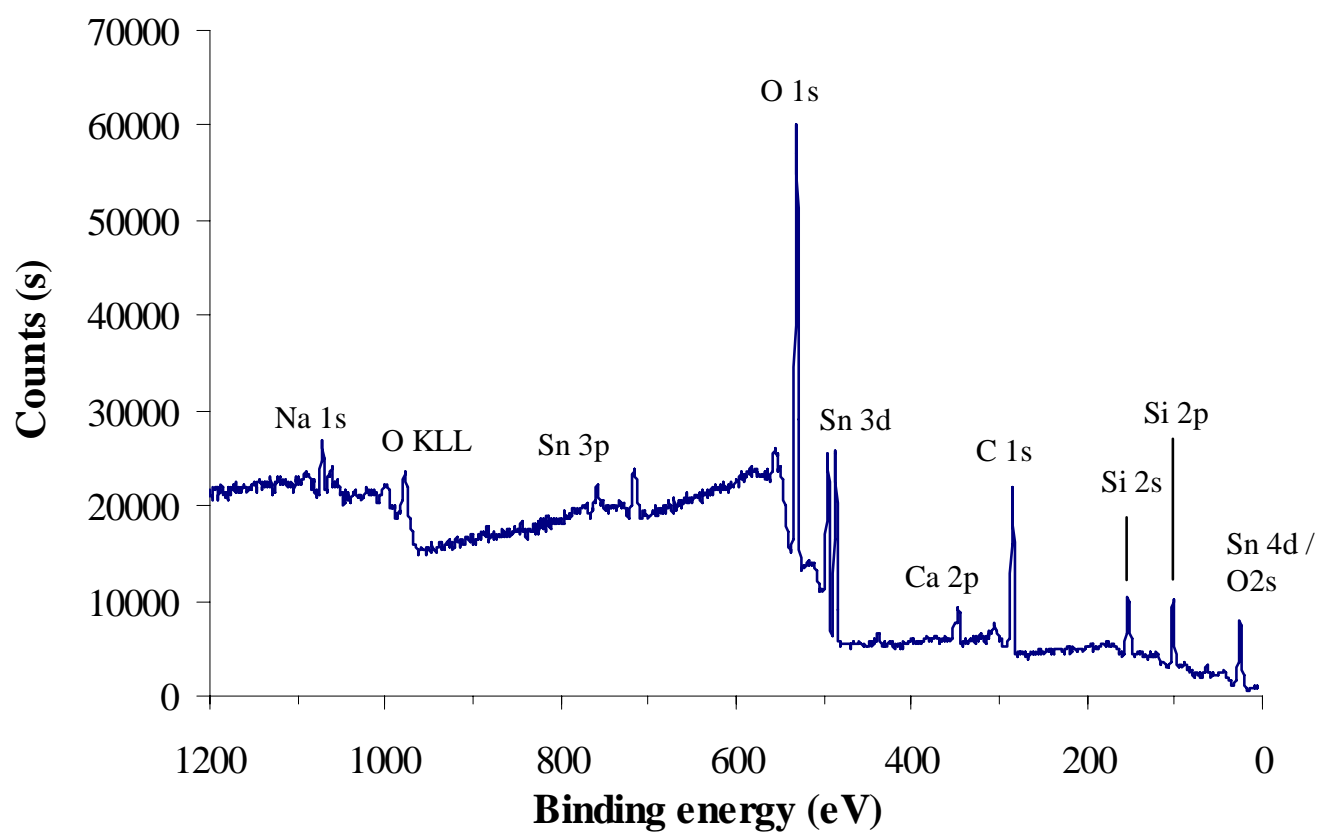

Figure 4. XPS survey spectra of the ultrasonically-cleaned samples for (a) side 1 and (b) side 2 . 
(a)

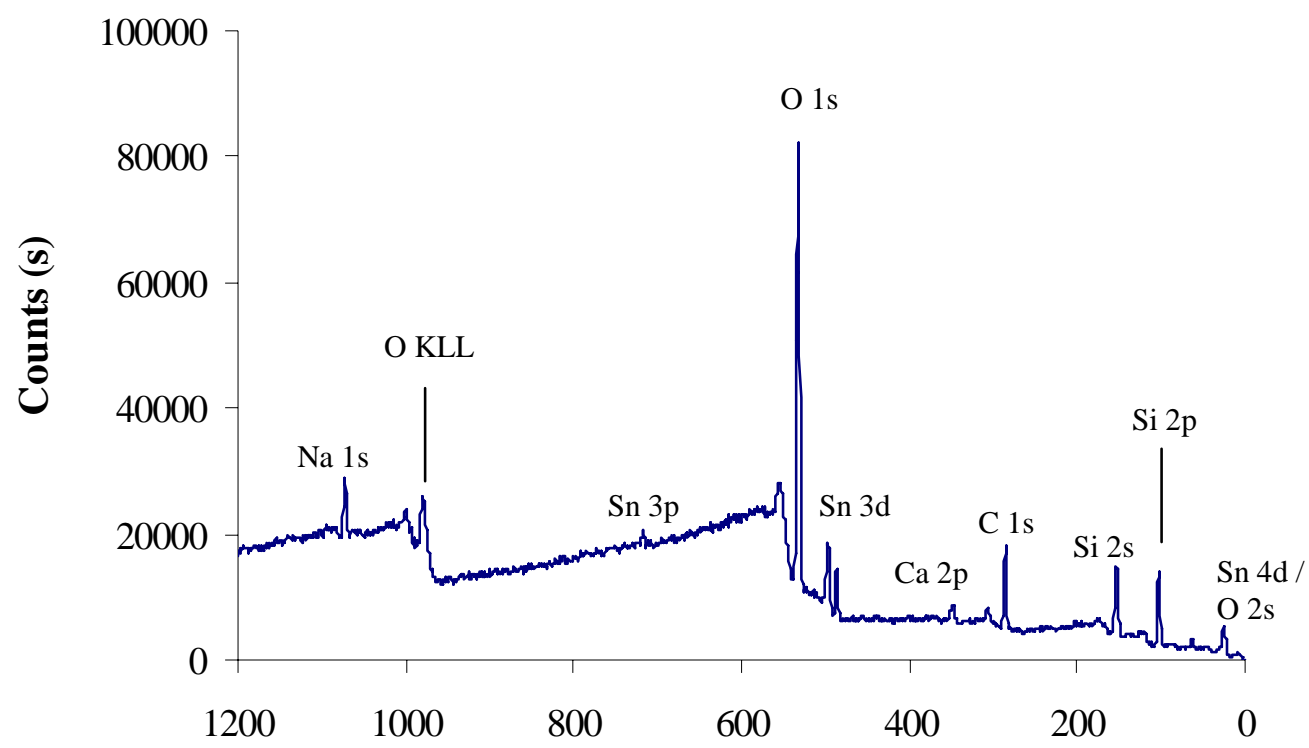

Binding energy (eV)

(b)

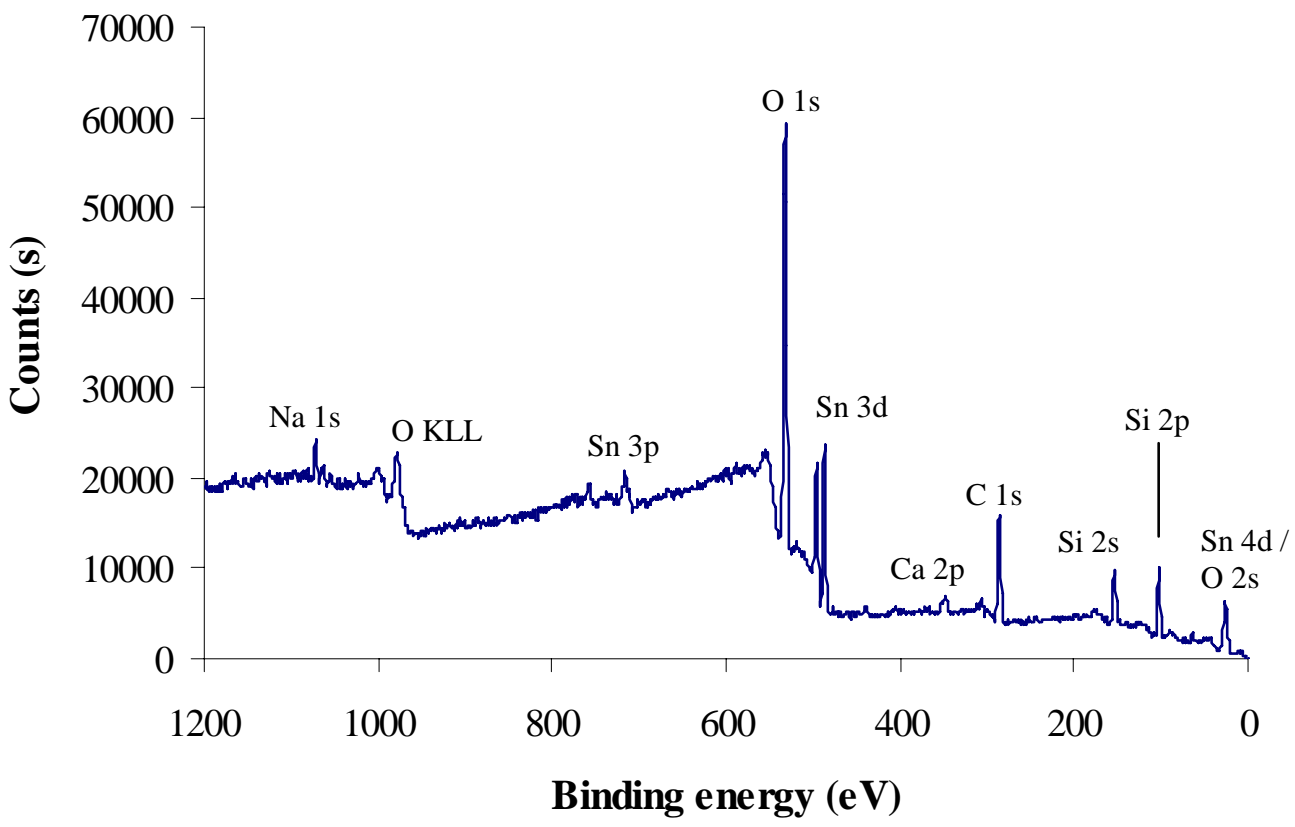

Figure 5. XPS survey spectra of the GPS-pretreated samples for (a) side 1 and (b) side 2. 
(a)

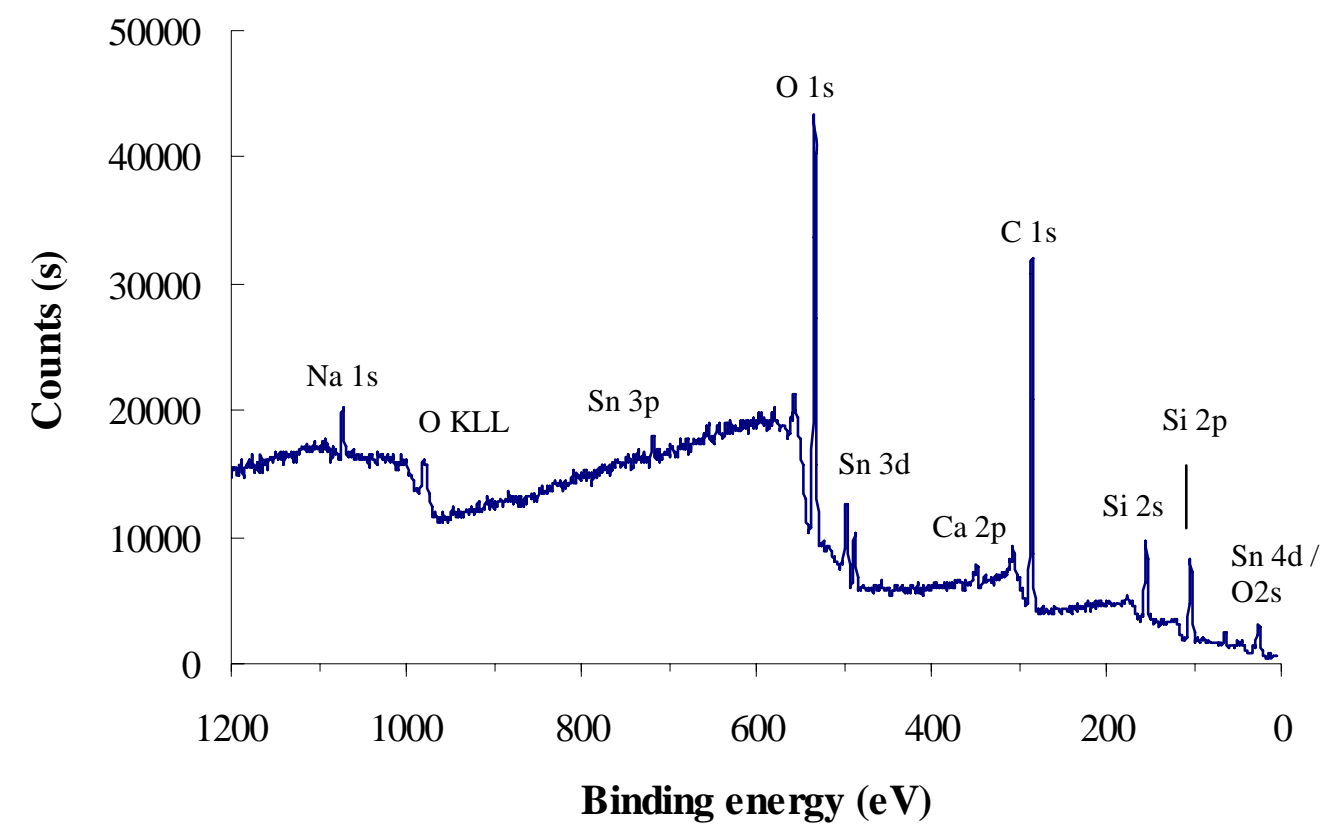

(b)

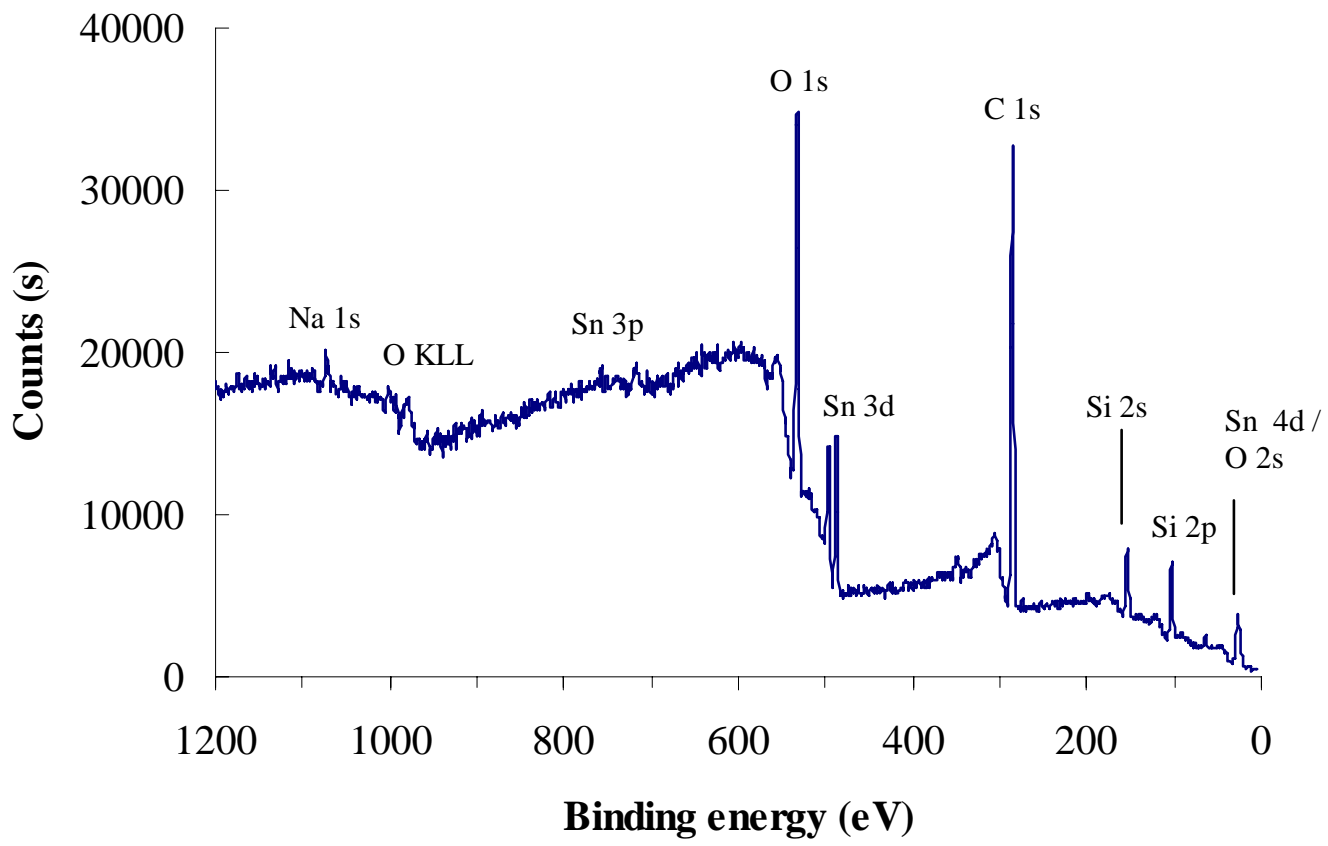

Figure 6. XPS survey spectra of the VTS pretreated samples for (a) side 1 and (b) side 2. 
(a)

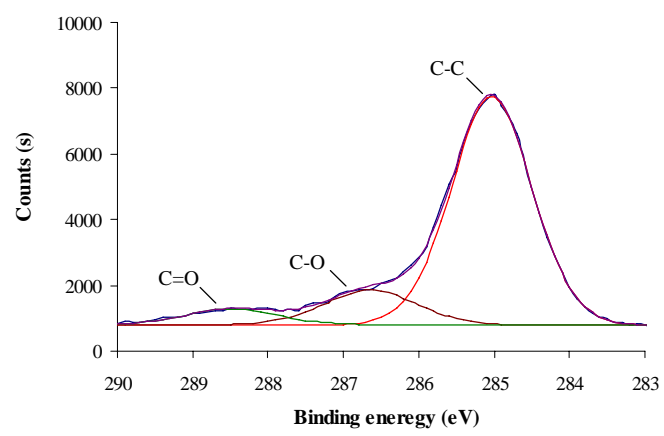

(b)

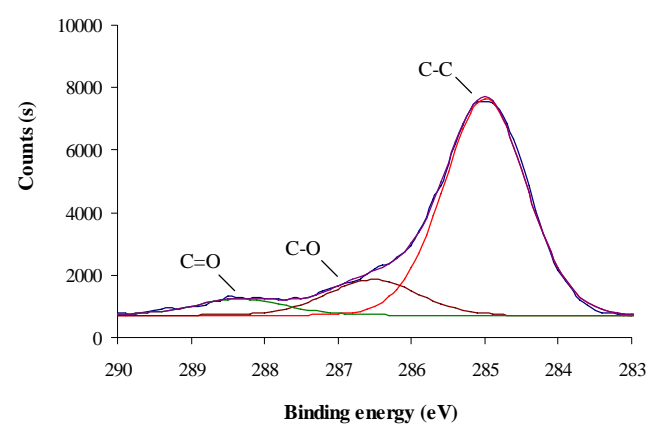

Figure 7. High-resolution XPS spectra of the peak-fitted C1s signal for the ultrasonicallycleaned glass; (a) side 1 and (b) side 2.

(a)

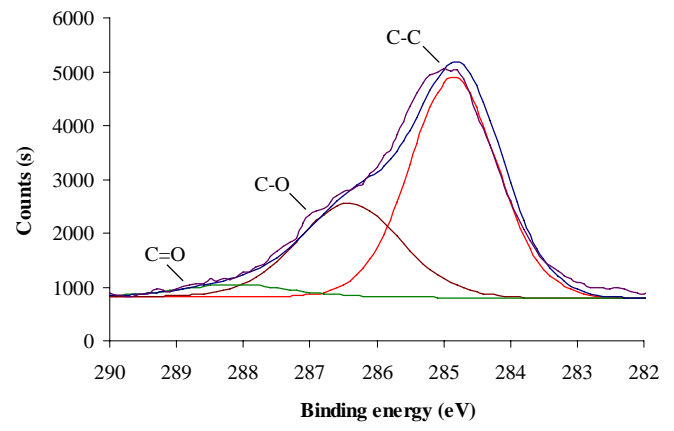

(b)

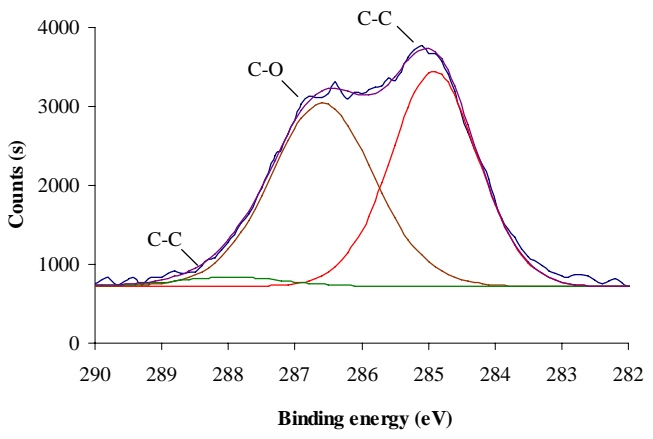

Figure 8. High-resolution XPS spectra of the peak-fitted C1s signal for the GPS-pretreated glass; (a) side 1 and (b) side 2.

(a)

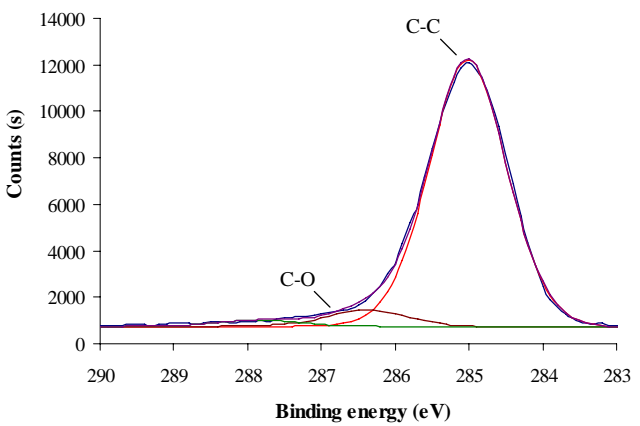

(b)

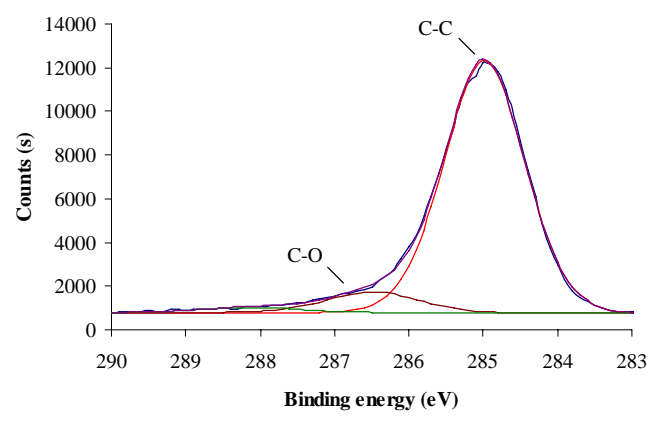

Figure 9. High-resolution XPS spectra of the peak-fitted C1s signal for the vinyl-terminated long carbon-chain silane (VTS)-pretreated glass; (a) side 1 and (b) side 2. 


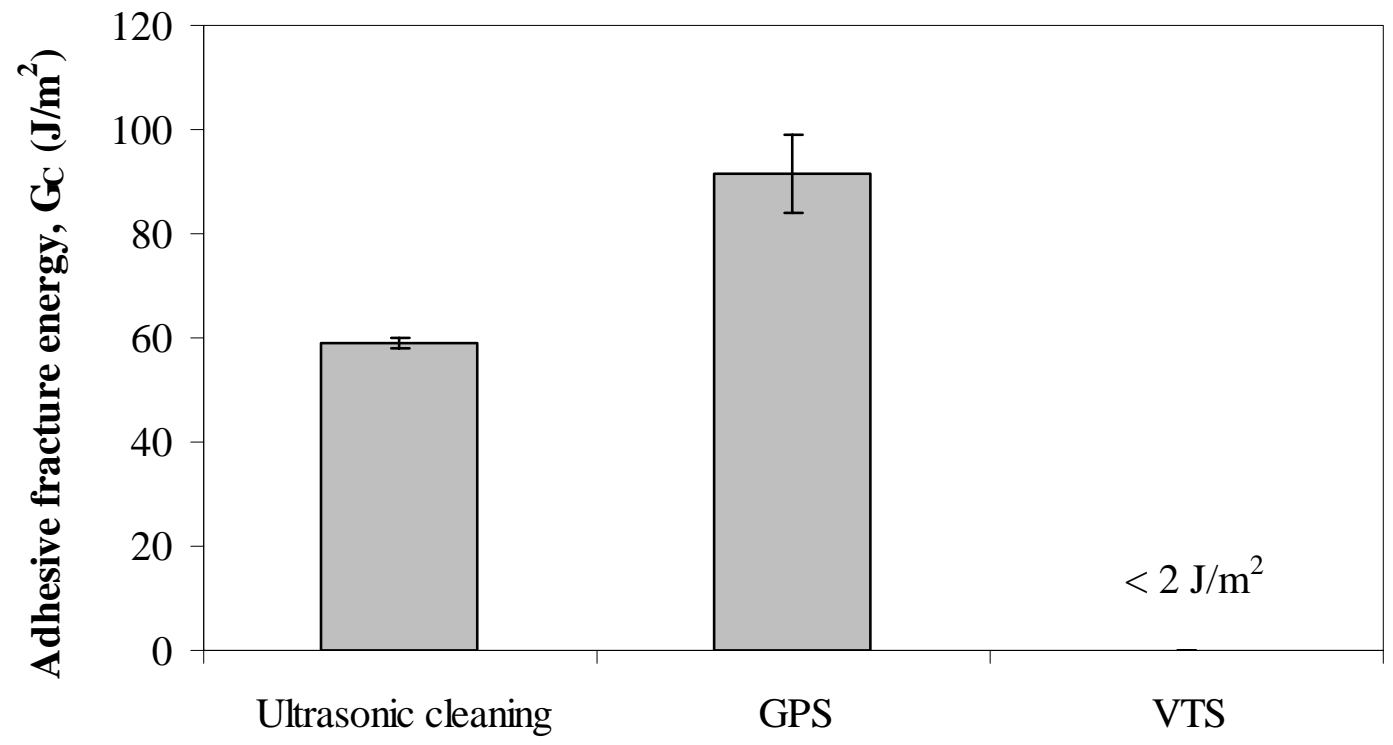

Figure 10. Values of the adhesive fracture energy, $G_{C}$, for various types of the glass/epoxy/glass DCB joints tested in an aqueous environment. The crack velocity for these tests was $0.3 \mathrm{~mm} / \mathrm{min}$. The error bars represent the standard deviations. 